\title{
Faber Polynomial Coefficient Estimates for a Comprehensive Subclass of Analytic Bi-Univalent Functions Defined by Subordination
}

\author{
Ahmad Zireh* \\ E. Analouei Adegani \\ Serap Bulut
}

\begin{abstract}
In this paper, we find coefficient estimates by a new method making use of the Faber polynomial expansions for a comprehensive subclass of analytic bi-univalent functions, which is defined by subordinations in the open unit disk. The coefficient bounds presented in this paper would generalize and improve some recent works appeared in the literature.
\end{abstract}

\section{Introduction}

Let $\mathbb{C}$ be the set of complex numbers, $\mathbb{N}:=\{1,2, \cdots\}$ and $\mathbb{Z}:=\{0, \pm 1, \pm 2, \cdots\}$. Let $\mathcal{A}$ denote the class of all functions of the form

$$
f(z)=z+\sum_{n=2}^{\infty} a_{n} z^{n},
$$

which are analytic in the open unit disk

$$
\mathbb{U}=\{z: z \in \mathbb{C} \text { and }|z|<1\} .
$$

*Corresponding author.

Received by the editors in January 2016 - In revised form in April 2016.

Communicated by G. Godefroy.

2010 Mathematics Subject Classification : 30C45, 30C80.

Key words and phrases : Bi-univalent functions, Coefficient estimates, Univalent functions, Faber polynomial, Subordination. 
Denote by $\mathcal{S}$ the class of all functions in the normalized analytic function class $\mathcal{A}$ which are univalent in $\mathbb{U}$.

Since univalent functions are one-to-one, they are invertible and the inverse functions need not be defined on the entire unit disk $\mathbb{U}$. In fact, the Koebe onequarter theorem [10] ensures that the image of $\mathbb{U}$ under every univalent function $f \in \mathcal{S}$ contains a disk of radius $1 / 4$. Thus every function $f \in \mathcal{S}$ has an inverse $f^{-1}$, which is defined by

$$
f^{-1}(f(z))=z \quad(z \in \mathbb{U})
$$

and

$$
f\left(f^{-1}(w)\right)=w \quad\left(|w|<r_{0}(f) ; r_{0}(f) \geq \frac{1}{4}\right) .
$$

In fact, the inverse function $f^{-1}$ is given by

$$
f^{-1}(w)=w-a_{2} w^{2}+\left(2 a_{2}^{2}-a_{3}\right) w^{3}-\left(5 a_{2}^{3}-5 a_{2} a_{3}+a_{4}\right) w^{4}+\cdots .
$$

A function $f \in \mathcal{S}$ is said to be bi-univalent in $\mathbb{U}$ if both $f$ and $f^{-1}$ are univalent in $\mathbb{U}$. Let $\Sigma$ denote the class of bi-univalent functions in $\mathbb{U}$ given by (1.1).

Determination of the bounds for the coefficients $a_{n}$ is an important problem in geometric function theory as they give information about the geometric properties of these functions.

Lewin [21] investigated the class $\Sigma$ of bi-univalent functions and showed that $\left|a_{2}\right|<1.51$ for the functions belonging to $\Sigma$. Subsequently, Brannan and Clunie [6] conjectured that $\left|a_{2}\right| \leq \sqrt{2}$. Kedzierawski [19] proved this conjecture for a special case when the function $f$ and $f^{-1}$ are starlike functions. Recently, Srivastava et al. [24], Frasin and Aouf [12], and Ali et al. [4] found estimates for the first two coefficients of certain subclasses of bi-univalent functions. Not much is known about the higher coefficients of bi-univalent functions; in fact Ali et al. [4] remarked that finding the bounds for $\left|a_{n}\right|(n \in \mathbb{N}-\{1,2\})$ for the bi-univalent functions is an open problem.

Recently, Bulut [8] introduced a comprehensive subclass $\mathcal{H}_{\Sigma}^{\lambda, \mu}(\varphi, \Theta)$ of analytic bi-univalent functions and obtained estimates on the coefficients $\left|a_{2}\right|$ and $\left|a_{3}\right|$ for functions in this subclass. In this paper, we use the Faber polynomial expansions to obtain estimates of coefficients $\left|a_{n}\right|$ where $n \geq 3$, of functions in these subclasses. Consequently, we obtain improvements on the bounds found by Bulut [8] for the first two coefficients $\left|a_{2}\right|$ and $\left|a_{3}\right|$ of functions in this subclass.

\section{Preliminary results}

Some special classes of univalent functions play an important role in geometric function theory because of their geometric properties, such as, the classes of convex, starlike, strongly convex and strongly starlike functions. It is fairly common that a function in one of these classes is lying in a given region in the right half-plan and the region is often symmetric with respect to the real axis. In this 
section we define a subclass $\mathcal{H}_{\Sigma}^{\lambda, \mu}(\varphi ; \Theta)$ (Definition 2.1), which in particular case (for $\lambda=1, \mu=0$ and $\left.\Theta=\frac{z}{1-z}\right)$ reduces to class starlike functions $\left(z f^{\prime}(z) / f(z)\right.$ ). Therefore in this paper, we assume that $\varphi$ is an analytic function with positive real part in the unit disk $\mathbb{U}$ and $\varphi(\mathbb{U})$ is symmetric with respect to the real axis, satisfying $\varphi(0)=1, \varphi^{\prime}(0)>0$ such that it has series expansion of the form

$$
\varphi(z)=1+B_{1} z+B_{2} z^{2}+B_{3} z^{3}+\cdots \quad\left(B_{1}>0\right) .
$$

Denote by $f * \Theta$ the Hadamard product (or convolution) of the functions $f$ and $\Theta$, that is, if $f(z)=z+\sum_{n=2}^{\infty} a_{n} z^{n}$ and $\Theta(z)=z+\sum_{n=2}^{\infty} b_{n} z^{n}$, then

$$
(f * \Theta)(z)=z+\sum_{n=2}^{\infty} a_{n} b_{n} z^{n} .
$$

For two functions $f$ and $F$ which are analytic in $\mathbb{U}$, we say that the function $f$ is subordinate to $F$ in $\mathbb{U}$, and write

$$
f(z) \prec F(z) \quad(z \in \mathbb{U}),
$$

if there exists a Schwarz function $\omega$, which is analytic in $\mathbb{U}$ with

$$
\omega(0)=0 \quad \text { and } \quad|\omega(z)|<1, z \in \mathbb{U},
$$

such that

$$
f(z)=F(\omega(z)), \quad z \in \mathbb{U} .
$$

By Schwarz's lemma we have $|\omega(z)| \leq|z|$, which concludes $\omega(\mathbb{U}) \subset \mathbb{U}$. Since $\omega(0)=0$ and $\omega(\mathbb{U}) \subset \mathbb{U}$ it follows that

if $f(z) \prec F(z)$ then $f(0)=F(0)$ and $f(\mathbb{U}) \subset F(\mathbb{U})$.

Furthermore, if the function $F$ is univalent in $\mathbb{U}$, then we have the following equivalence

$$
f(z) \prec F(z) \quad(z \in \mathbb{U}) \Leftrightarrow f(0)=F(0) \quad \text { and } \quad f(\mathbb{U}) \subset F(\mathbb{U}) .
$$

Recently, Bulut [8] introduced a comprehensive subclass of analytic bi-univalent functions and obtained non-sharp estimates on the coefficients $\left|a_{2}\right|$ and $\left|a_{3}\right|$ for functions in this subclass as follow.

Definition 2.1. [8] Let the function $f$, defined by (1.1), be in the class $\mathcal{A}$ and let

$$
\Theta \in \Sigma \quad \text { and } \quad \Theta(z)=z+\sum_{n=2}^{\infty} b_{n} z^{n} \quad,\left(b_{n}>0\right) \text {. }
$$

We say that

$$
f \in \mathcal{H}_{\Sigma}^{\lambda, \mu}(\varphi ; \Theta) \quad(\lambda \geq 1, \mu \geq 0),
$$

if the following conditions are satisfied:

$$
\begin{gathered}
f \in \Sigma, \\
(1-\lambda)\left(\frac{(f * \Theta)(z)}{z}\right)^{\mu}+\lambda(f * \Theta)^{\prime}(z)\left(\frac{(f * \Theta)(z)}{z}\right)^{\mu-1} \prec \varphi(z) \quad,(z \in \mathbb{U})
\end{gathered}
$$


and

$$
\begin{array}{r}
(1-\lambda)\left(\frac{(f * \Theta)^{-1}(w)}{w}\right)^{\mu}+\lambda\left((f * \Theta)^{-1}\right)^{\prime}(w)\left(\frac{(f * \Theta)^{-1}(w)}{w}\right)^{\mu-1} \\
\prec \varphi(w), \\
(w \in \mathbb{U}),
\end{array}
$$

where the function $(f * \Theta)^{-1}$ is given by

$$
\begin{aligned}
(f * \Theta)^{-1}(w)=w-a_{2} b_{2} w^{2}+ & \left(2 a_{2}^{2} b_{2}^{2}-a_{3} b_{3}\right) w^{3}- \\
& \left(5 a_{2}^{3} b_{2}^{3}-5 a_{2} b_{2} a_{3} b_{3}+a_{4} b_{4}\right) w^{4}+\cdots .
\end{aligned}
$$

Theorem 2.2. [8] Let the function $f(z)$ given by the Taylor-Maclaurin series expansion (1.1) be in the function class

$$
\mathcal{H}_{\Sigma}^{\lambda, \mu}(\varphi ; \Theta) \quad(\lambda \geq 1, \mu \geq 0)
$$

with $\Theta$ is given by (2.2). Then

$$
\begin{aligned}
\left|a_{2}\right| \leq & \frac{1}{b_{2}} \min \left\{\frac{B_{1}}{\lambda+\mu}, \sqrt{\frac{2\left(B_{1}+\left|B_{2}-B_{1}\right|\right)}{(\mu+1)(2 \lambda+\mu)}}\right. \\
& \left.\frac{B_{1} \sqrt{2 B_{1}}}{\sqrt{\left|B_{1}^{2}(\mu+1)(2 \lambda+\mu)-2\left(B_{2}-B_{1}\right)(\lambda+\mu)^{2}\right|}}\right\},
\end{aligned}
$$

and

$$
\begin{aligned}
& \left|a_{3}\right| \leq \frac{1}{b_{3}} \min \left\{\frac{B_{1}^{2}}{(\lambda+\mu)^{2}}+\frac{B_{1}}{2 \lambda+\mu}, \frac{B_{1}[(\mu+3)+|1-\mu|]}{2(\mu+1)(2 \lambda+\mu)}+\frac{2\left|B_{2}-B_{1}\right|}{(\mu+1)(2 \lambda+\mu)},\right. \\
& \left.\frac{2 B_{1}^{3}}{\left|B_{1}^{2}(\mu+1)(2 \lambda+\mu)-2\left(B_{2}-B_{1}\right)(\lambda+\mu)^{2}\right|}+\frac{B_{1}}{2 \lambda+\mu}\right\} \text {. }
\end{aligned}
$$

In the present paper by using the Faber polynomial expansions we obtain estimates of coefficients $\left|a_{n}\right|$ where $n \geq 3$, of functions in the subclasses $\mathcal{H}_{\Sigma}^{\lambda, \mu}(\varphi ; \Theta)$. The Faber polynomials introduced by Faber [13] play an important role in various areas of mathematical sciences, especially in geometric function theory. Several authors worked on using Faber polynomial expansions to find coefficient estimates for classes bi-univalent functions, see for example [8, 14, 15, 16, 17, 18]. For this purpose we need the following lemmas. 
Lemma 2.3. Let $\phi(z)=a_{2} z+a_{3} z^{2}+\cdots+a_{k+1} z^{k}+\cdots$. Then for any $m \in \mathbb{N}$, the coefficient of $z^{n}$ in $(\phi(z))^{m}$ is equal to

$$
D_{n}^{m}\left(a_{2}, a_{3}, \cdots a_{n+1}\right)=\sum \frac{m !\left(a_{2}\right)^{i_{1}} \cdots\left(a_{n+1}\right)^{i_{n}}}{i_{1} ! \cdots i_{n} !}
$$

where the sum is taken over all nonnegative integers $i_{1}, \cdots, i_{n}$ satisfying

$$
\left\{\begin{array}{l}
i_{1}+i_{2}+\cdots+i_{n}=m \\
i_{1}+2 i_{2}+\cdots+n i_{n}=n
\end{array}\right.
$$

It is clear that

$$
D_{n}^{n}\left(a_{2}, a_{3}, \cdots a_{n+1}\right)=a_{2}^{n}
$$

Proof. It is derived from the expansion $\left(a_{2} z+a_{3} z^{2}+\cdots+a_{n+1} z^{n}\right)^{m}$.

Lemma 2.4. $[1,2]$ Let $f(z)=z+\sum_{n=2}^{\infty} a_{n} z^{n} \in \mathcal{S}$. Then for any $p \in \mathbb{Z}$, there are the polynomials $K_{n}^{p}$, such that

$$
\left(1+a_{2} z+a_{3} z^{2}+\cdots+a_{k} z^{k-1}+\cdots\right)^{p}=1+\sum_{n=1}^{\infty} K_{n}^{p}\left(a_{2}, a_{3}, \cdots, a_{n+1}\right) z^{n},
$$

where

$K_{n}^{p}\left(a_{2}, \cdots, a_{n+1}\right)=p a_{n+1}+\frac{p(p-1)}{2} D_{n}^{2}+\frac{p !}{(p-3) ! 3 !} D_{n}^{3}+\cdots+\frac{p !}{(p-n) !(n) !} D_{n}^{n}$,

with $D_{n}^{m}$ is given by (2.5). In particular, $K_{n}^{1}=a_{n+1}, K_{1}^{2}=2 a_{2}, K_{2}^{2}=2 a_{3}+a_{2}^{2}$, $K_{3}^{2}=2 a_{4}+2 a_{2} a_{3}, K_{4}^{2}=2 a_{5}+2 a_{2} a_{4}+a_{3}^{2}$.

Lemma 2.5. Let $f(z)=z+\sum_{n=2}^{\infty} a_{n} z^{n} \in \mathcal{S}$, then for every $\mu \geq 0$, we have the following expansion,

$$
\begin{aligned}
(1-\lambda)\left(\frac{f(z)}{z}\right)^{\mu}+ & \lambda f^{\prime}(z)\left(\frac{f(z)}{z}\right)^{\mu-1} \\
=1 & +\sum_{n \geq 1}\left(\frac{\mu+\lambda n}{\mu}\right) K_{n}^{\mu}\left(a_{2}, a_{3}, \cdots, a_{n+1}\right) z^{n}
\end{aligned}
$$

where

$$
\begin{aligned}
& \left(\frac{\mu+\lambda n}{\mu}\right) K_{n}^{\mu}\left(a_{2}, a_{3}, \cdots, a_{n+1}\right)=[\mu+\lambda n](\mu-1) ! \\
& \times\left[\sum_{i_{1}+2 i_{2}+\cdots+n i_{n}=n} \frac{\left(a_{2}\right)^{i_{1}}\left(a_{3}\right)^{i_{2}} \cdots\left(a_{n+1}\right)^{i_{n}}}{i_{1} ! i_{2} ! \cdots i_{n} !\left[\mu-\left(i_{1}+i_{2}+\cdots+i_{n}\right)\right] !}\right] .
\end{aligned}
$$

In particular, the first two terms are, $\left(\frac{\mu+\lambda}{\mu}\right) K_{1}^{\mu}\left(a_{2}\right)=(\mu+\lambda) a_{2},\left(\frac{\mu+2 \lambda}{\mu}\right) K_{2}^{\mu}\left(a_{2}, a_{3}\right)=$ $(\mu+2 \lambda)\left[\frac{\mu-1}{2} a_{2}^{2}+a_{3}\right]$. 
Proof. For $f(z)=z+\sum_{n=2}^{\infty} a_{n} z^{n}$, consider $h(z)=\frac{f(z)}{z}=1+\sum_{n \geq 1} a_{n+1} z^{n}$, then by using Lemma 2.4, we have $h(z)^{\mu}=1+\sum_{n \geq 1} K_{n}^{\mu}\left(a_{2}, a_{3}, \cdots, a_{n+1}\right) z^{n}$, therefore

$$
\begin{aligned}
& (1-\lambda)\left(\frac{f(z)}{z}\right)^{\mu}+\lambda f^{\prime}(z)\left(\frac{f(z)}{z}\right)^{\mu-1}=h(z)^{\mu}+\frac{\lambda z}{\mu} \frac{d}{d z}\left(h(z)^{\mu}\right) \\
& =1+\sum_{n \geq 1}\left(\frac{\mu+\lambda n}{\mu}\right) K_{n}^{\mu}\left(a_{2}, a_{3}, \cdots, a_{n+1}\right) z^{n} .
\end{aligned}
$$

Now by using equation (2.6), we have the result.

Corollary 2.6. Let $f \in \mathcal{H}_{\Sigma}^{\lambda, \mu}(\varphi ; \Theta)$, then we have the following expansion

$$
\begin{aligned}
&(1-\lambda)\left(\frac{f * \Theta(z)}{z}\right)^{\mu}+ \lambda(f * \Theta)^{\prime}(z)\left(\frac{f * \Theta(z)}{z}\right)^{\mu-1} \\
&=1+\sum_{n=2}^{\infty} F_{n-1}\left(a_{2} b_{2}, a_{3} b_{3}, \cdots, a_{n} b_{n}\right) z^{n-1}
\end{aligned}
$$

where

$$
\begin{aligned}
& F_{n-1}\left(a_{2} b_{2}, a_{3} b_{3}, \cdots, a_{n} b_{n}\right)=\left(\frac{\mu+(n-1) \lambda}{\mu}\right) K_{n-1}^{\mu}\left(a_{2} b_{2}, a_{3} b_{3}, \cdots, a_{n} b_{n}\right) \\
& =[\mu+(n-1) \lambda](\mu-1) ! \times \\
& \left.\qquad \sum_{i_{1}+\cdots+(n-1) i_{n-1}=n-1} \frac{\left(a_{2} b_{2}\right)^{i_{1}}\left(a_{3} b_{3}\right)^{i_{2}} \cdots\left(a_{n} b_{n}\right)^{i_{n-1}}}{i_{1} ! i_{2} ! \cdots i_{n-1} !\left[\mu-\left(i_{1}+i_{2}+\cdots+i_{n-1}\right)\right] !}\right] .
\end{aligned}
$$

Proof. Since $(f * \Theta)(z)=z+\sum_{n \geq 1} a_{n} b_{n} z^{n}$, by applying Lemma 2.5 we have the result.

Lemma 2.7. Let $f(z)=z+\sum_{n=2}^{\infty} a_{n} z^{n} \in \mathcal{S}$. Then for every $\mu \geq 0,\left(f^{-1}\right)^{\mu}$, is given by the expansion,

$$
\left(f^{-1}(w)\right)^{\mu}=w^{\mu}+\sum_{n \geq 1} \frac{\mu}{\mu+n} K_{n}^{-(n+\mu)} w^{n+\mu} .
$$

Proof. Let $\left(\frac{f^{-1}(w)}{w}\right)^{\mu}=1+\sum_{n \geq 1} c_{n} w^{n}$, then by Cauchy theorem,

$$
c_{n}=\frac{1}{2 i \pi} \int_{\gamma} \frac{f^{-1}(w)^{\mu}}{w^{\mu} w^{n+1}} d w=\frac{1}{2 i \pi} \int \frac{z^{\mu} f^{\prime}(z)}{f(z)^{\mu+n+1}} d z \text {, where } w=f(z) .
$$

Integrate by parts, then

$$
c_{n}=\frac{1}{2 i \pi} \int \frac{\mu}{\mu+n} \times \frac{f(z)^{-(n+\mu)}}{z^{-(n+\mu)}} \times \frac{1}{z^{n+1}} d z=\frac{\mu}{\mu+n} K_{n}^{-(n+\mu)} .
$$

Consequently, the expansion of $\left(\frac{f^{-1}(w)}{w}\right)^{\mu}$, is given by

$$
\left(\frac{f^{-1}(w)}{w}\right)^{\mu}=1+\sum_{n \geq 1} \frac{\mu}{\mu+n} K_{n}^{-(n+\mu)} w^{n}
$$


If we take $\mu=1 \mathrm{in}$ Lemma 2.7 , we have the following result.

Corollary 2.8. [2] Let $f(z)=z+\sum_{n=2}^{\infty} a_{n} z^{n} \in \mathcal{S}$. Then the inverse map of $f$, is given by the expansion,

$$
f^{-1}(w)=w+\sum_{n=2}^{\infty} \frac{1}{n} K_{n-1}^{-n}\left(a_{2}, a_{3}, \cdots, a_{n}\right) w^{n} .
$$

In particular, the first three terms of $K_{n-1}^{-n}$ are

$$
\frac{1}{2} K_{1}^{-2}=-a_{2}, \quad \frac{1}{3} K_{2}^{-3}=2 a_{2}^{2}-a_{3}, \quad \frac{1}{4} K_{3}^{-4}=-\left(5 a_{2}^{3}-5 a_{2} a_{3}+a_{4}\right) .
$$

Corollary 2.9. Let $f(z)=z+\sum_{n=2}^{\infty} a_{n} z^{n} \in \mathcal{S}$, then for every $\mu \geq 0$, we have the following expansion,

$$
\begin{aligned}
&(1-\lambda)\left(\frac{f^{-1}(w)}{w}\right)^{\mu}+ \lambda\left(f^{-1}\right)^{\prime}(w)\left(\frac{f^{-1}(w)}{w}\right)^{\mu-1} \\
&=1+\sum_{n \geq 1} \frac{\mu+\lambda n}{\mu+n} K_{n}^{-(n+\mu)} w^{n}
\end{aligned}
$$

Proof. Let $f^{-1}(w)=w h_{1}(w)$, then $\left(f^{-1}\right)^{\prime}(w)=h_{1}(w)+w h_{1}^{\prime}(w)$. Now by Lemma 2.7, we have $\left(h_{1}(w)\right)^{\mu}=1+\sum_{n \geq 1} \frac{\mu}{\mu+n} K_{n}^{-(n+\mu)} w^{n}$. Therefore

$$
\begin{aligned}
(1-\lambda)\left(\frac{f^{-1}(w)}{w}\right)^{\mu}+ & \lambda\left(f^{-1}\right)^{\prime}(w)\left(\frac{f^{-1}(w)}{w}\right)^{\mu-1} \\
& =(1-\lambda)\left(h_{1}(w)\right)^{\mu}+\lambda\left(h_{1}(w)+w h_{1}^{\prime}(w)\right)\left(h_{1}(w)\right)^{\mu-1} \\
& =\left(h_{1}(w)\right)^{\mu}+\lambda w h_{1}^{\prime}(w)\left(h_{1}(w)\right)^{\mu-1} \\
& =\left(h_{1}(w)\right)^{\mu}+\frac{\lambda w}{\mu} \frac{d}{d w}\left(\left(h_{1}(w)\right)^{\mu}\right) \\
& =1+\sum_{n \geq 1} \frac{\mu+\lambda n}{\mu+n} K_{n}^{-(n+\mu)} w^{n} .
\end{aligned}
$$

Corollary 2.10. Let $f(z)=z+\sum_{n=2}^{\infty} a_{n} z^{n} \in \mathcal{S}$, then for every $\mu \geq 0$ and $n \in \mathbb{N}$ we have

$$
\frac{\mu}{\mu+n} K_{n}^{-(n+\mu)}\left(a_{2}, \cdots, a_{n+1}\right)=K_{n}^{\mu}\left(L_{2}, \cdots, L_{n+1}\right),
$$

where $L_{n}=\frac{1}{n} K_{n-1}^{-n}\left(a_{2}, a_{3}, \cdots, a_{n}\right)$.

Proof. From equation (2.10) we have

$$
\left(\frac{f^{-1}(w)}{w}\right)=1+\sum_{n \geq 2}\left(\frac{1}{n} K_{n-1}^{-n}\left(a_{2}, a_{3}, \cdots, a_{n}\right)\right) w^{n-1} .
$$

Therefore by using Lemma 2.4, we have

$$
\left(\frac{f^{-1}(w)}{w}\right)^{\mu}=1+\sum_{n \geq 1} K_{n}^{\mu}\left(L_{2}, \cdots, L_{n+1}\right) w^{n},
$$


where $L_{n}=\frac{1}{n} K_{n-1}^{-n}\left(a_{2}, a_{3}, \cdots, a_{n}\right)$.

Comparing the corresponding coefficients of equations (2.9) and (2.11), we have the result.

Lemma 2.11. Let $f \in \mathcal{H}_{\Sigma}^{\lambda, \mu}(\varphi ; \Theta)$, then we have the following expansion

$$
\begin{aligned}
& (1-\lambda)\left(\frac{(f * \Theta)^{-1}(w)}{w}\right)^{\mu}+\lambda\left((f * \Theta)^{-1}\right)^{\prime}(w)\left(\frac{(f * \Theta)^{-1}(w)}{w}\right)^{\mu-1} \\
= & 1+\sum_{n=2}^{\infty} F_{n-1}\left(A_{2}, A_{3}, \cdots, A_{n}\right) w^{n-1}
\end{aligned}
$$

where $F_{n-1}=\left(\frac{\mu+(n-1) \lambda}{\mu}\right) K_{n-1}^{\mu}$ and $A_{n}=\frac{1}{n} K_{n-1}^{-n}\left(a_{2} b_{2}, a_{3} b_{3}, \cdots, a_{n} b_{n}\right)$.

Proof. By applying Corollary 2.9 for $(f * \Theta)(z)=z+\sum_{n \geq 2} a_{n} b_{n} z^{n}$ and using Corollary 2.10, we have the result.

Lemma 2.12. Assume that $u(z)$ and $v(z)$ are analytic in the unit disk $\mathbb{U}$ with $u(0)=$ $v(0)=0,|u(z)|<1,|v(z)|<1$, and suppose that

$$
u(z)=z\left(p_{1}+\sum_{n=2}^{\infty} p_{n} z^{n-1}\right) \quad \text { and } \quad v(z)=z\left(q_{1}+\sum_{n=2}^{\infty} q_{n} z^{n-1}\right) \quad(z \in \mathbb{U}) .
$$

Then

$$
\left|p_{1}\right| \leq 1, \quad\left|p_{n}\right| \leq 1-\left|p_{1}\right|^{2}, \quad\left|q_{1}\right| \leq 1, \quad\left|q_{n}\right| \leq 1-\left|q_{1}\right|^{2}, \quad n=2,3, \cdots .
$$

Proof. For proof see [22, Page 172].

Lemma 2.13. Let $f \in \mathcal{H}_{\Sigma}^{\lambda, \mu}(\varphi ; \Theta)$. Then

$$
F_{n-1}\left(a_{2} b_{2}, a_{3} b_{3}, \cdots, a_{n} b_{n}\right)=\sum_{k=1}^{n-1} B_{k} D_{n-1}^{k}\left(p_{1}, p_{2}, \cdots, p_{n-1}\right),
$$

and

$$
F_{n-1}\left(A_{2}, A_{3}, \cdots, A_{n}\right)=\sum_{k=1}^{n-1} B_{k} D_{n-1}^{k}\left(q_{1}, q_{2}, \cdots, q_{n-1}\right),
$$

where $F_{n-1}=\left(\frac{\mu+(n-1) \lambda}{\mu}\right) K_{n-1}^{\mu}$ and $A_{n}=\frac{1}{n} K_{n-1}^{-n}\left(a_{2} b_{2}, a_{3} b_{3}, \cdots, a_{n} b_{n}\right)$.

Proof. By Definition 2.1, since $f \in \mathcal{H}_{\Sigma}^{\lambda, \mu}(\varphi ; \Theta)$, there are two Schwarz functions $u, v: \mathbb{U} \rightarrow \mathbb{U}$ with $u(0)=v(0)=0$, as in (2.13), so that

$$
(1-\lambda)\left(\frac{f * \Theta(z)}{z}\right)^{\mu}+\lambda(f * \Theta)^{\prime}(z)\left(\frac{f * \Theta(z)}{z}\right)^{\mu-1}=\varphi(u(z))
$$

and

$$
(1-\lambda)\left(\frac{(f * \Theta)^{-1}(w)}{w}\right)^{\mu}+\lambda\left((f * \Theta)^{-1}\right)^{\prime}(w)\left(\frac{(f * \Theta)^{-1}(w)}{w}\right)^{\mu-1}=\varphi(v(w)) .
$$


Now by using (2.1) and (2.5) we have,

$$
\begin{aligned}
\varphi(u(z)) & =1+B_{1} u(z)+B_{2}(u(z))^{2}+\cdots=1+B_{1} p_{1} z+\left(B_{1} p_{2}+B_{2} p_{1}^{2}\right) z^{2}+\cdots \\
& =1+\sum_{n=1}^{\infty} \sum_{k=1}^{n} B_{k} D_{n}^{k}\left(p_{1}, p_{2}, \cdots, p_{n}\right) z^{n},
\end{aligned}
$$

and

$$
\begin{aligned}
\varphi(v(w)) & =1+B_{1} v(z)+B_{2}(v(z))^{2}+\cdots=1+B_{1} q_{1} w+\left(B_{1} q_{2}+B_{2} q_{1}^{2}\right) w^{2}+\cdots \\
& =1+\sum_{n=1}^{\infty} \sum_{k=1}^{n} B_{k} D_{n}^{k}\left(q_{1}, q_{2}, \cdots, q_{n}\right) w^{n} .
\end{aligned}
$$

Comparing the corresponding coefficients of (2.8), (2.17) and (2.19) we get (2.15). Similarly, from (2.12), (2.18) and (2.20) we obtain (2.16).

Our first theorem introduces an upper bound for the coefficients $\left|a_{n}\right|$ of functions in the class $\mathcal{H}_{\Sigma}^{\lambda, \mu}(\varphi ; \Theta)$.

\section{Main Results}

In this section, first we obtain estimates of coefficients $\left|a_{n}\right|$ where $n \geq 3$, of analytic bi-univalent functions in the subclasses $\mathcal{H}_{\Sigma}^{\lambda, \mu}(\varphi ; \Theta)$. Next, we obtain estimates of coefficients $\left|a_{2}\right|$ and $\left|a_{3}\right|$ of functions in this subclass which are improvements on the bounds found by Bulut [8].

Theorem 3.1. For $\lambda \geq 1$ and $\mu \geq 0$, let $f \in \mathcal{H}_{\Sigma}^{\lambda, \mu}(\varphi ; \Theta)$ be given by (1.1). If $a_{k}=0$ for $2 \leq k \leq n-1$, then

$$
\left|a_{n}\right| \leq \frac{B_{1}}{[\mu+(n-1) \lambda] b_{n}}, \quad n \geq 3
$$

Proof. Note that $a_{k}=0$ for $2 \leq k \leq n-1$, so we have $A_{n}=-a_{n} b_{n}$ and $p_{1}=\cdots=$ $p_{n-2}=0, q_{1}=\cdots=q_{n-2}=0$. From (2.15)-(2.16), we have

$$
[\mu+(n-1) \lambda] a_{n} b_{n}=B_{1} p_{n-1}
$$

and

$$
-[\mu+(n-1) \lambda] a_{n} b_{n}=B_{1} q_{n-1} .
$$

Now, by solving either of the equations (3.2) and (3.3) for $a_{n}$ and applying $\left|p_{n-1}\right| \leq 1-\left|p_{1}\right|^{2} \leq 1,\left|q_{n-1}\right| \leq 1-\left|q_{1}\right|^{2} \leq 1$, it yields

$$
\begin{aligned}
\left|a_{n}\right| & =\frac{B_{1}\left|p_{n-1}\right|}{[\mu+(n-1) \lambda] b_{n}}=\frac{B_{1}\left|q_{n-1}\right|}{[\mu+(n-1) \lambda] b_{n}} \\
& \leq \frac{B_{1}}{[\mu+(n-1) \lambda] b_{n}},
\end{aligned}
$$

which gives the bound as asserted in (3.1) and this completes the proof. 
If we take $\Theta(z)=\frac{z}{1-z}$ and $\varphi(z)=\frac{1+(1-2 \beta) z}{1-z}=1+2(1-\beta) z+2(1-\beta) z^{2}+\cdots \quad(0 \leq \beta<1, z \in \mathbb{U})$ then, $B_{1}=B_{2}=2(1-\beta)$, in Theorem 3.1, and we have the following corollary which generalizes the results obtained by Bulut, see formula (27) in [8].

Corollary 3.2. For $\lambda \geq 1$ and $\mu \geq 0$, let $f \in \mathcal{H}_{\Sigma}^{\lambda, \mu}(\varphi ; \Theta)$ be given by (1.1). If $a_{k}=0$ for $2 \leq k \leq n-1$, then

$$
\left|a_{n}\right| \leq \frac{2(1-\beta)}{\mu+(n-1) \lambda}, \quad n \geq 3 .
$$

Remark 3.3. If we take $\lambda=1$ and $\mu=1$ in Corollary 3.2, the estimates of Jahangiri et al. [16, Theorem 1] and Jahangiri et al. [18, Theorem 2.1] are obtained.

Corollary 3.4. For $\lambda \geq 1, \mu \geq 0$ and $0<\alpha \leq 1$, let $f \in \mathcal{H}_{\Sigma}^{\lambda, \mu}\left(\left(\frac{1+z}{1-z}\right)^{\alpha} ; \Theta\right)$ be given by (1.1). If $a_{k}=0$ for $2 \leq k \leq n-1$, then

$$
\left|a_{n}\right| \leq \frac{2 \alpha}{[\mu+(n-1) \lambda] b_{n}}, \quad n \geq 3
$$

Proof. Since

$$
\varphi(z)=\left(\frac{1+z}{1-z}\right)^{\alpha}=1+2 \alpha z+2 \alpha^{2} z^{2}+\cdots
$$

then $B_{1}=2 \alpha$, now by using (3.1), we have result.

In what follows, we give refinements of inequalities (2.3) and (2.4) for class $\mathcal{H}_{\Sigma}^{\lambda, \mu}(\varphi ; \Theta)$.

Theorem 3.5. Let $f \in \mathcal{H}_{\Sigma}^{\lambda, \mu}(\varphi ; \Theta)$ be given by (1.1), $\lambda \geq 1$ and $\mu \geq 0$, with $\Theta$ is given by (2.2). Then

$$
\left|a_{2}\right| \leq \frac{1}{b_{2}} \min \left\{l(\lambda, \mu), \frac{B_{1} \sqrt{2 B_{1}}}{\sqrt{\left[2 B_{1}(\lambda+\mu)^{2}+\left|B_{1}^{2}(2 \lambda+\mu)(\mu+1)-2 B_{2}(\lambda+\mu)^{2}\right|\right]}}\right\},
$$

and

$$
\left|a_{3}\right| \leq \frac{1}{b_{3}} \min \{k(\lambda, \mu), h(\lambda, \mu)\}
$$

where

$$
l(\lambda, \mu)= \begin{cases}\sqrt{\frac{2\left|B_{1}\right|}{(2 \lambda+\mu)(\mu+1)}} & \left|B_{2}\right| \leq B_{1} \\ \sqrt{\frac{2\left|B_{2}\right|}{(2 \lambda+\mu)(\mu+1)}} & \left|B_{2}\right|>B_{1}\end{cases}
$$




$$
k(\lambda, \mu)= \begin{cases}\frac{B_{1}}{(2 \lambda+\mu)}, & B_{1} \leq \frac{(\lambda+\mu)^{2}}{2 \lambda+\mu} \\ \frac{B_{1}\left|B_{1}^{2}(2 \lambda+\mu)(\mu+1)-2 B_{2}(\lambda+\mu)^{2}\right|+2(2 \lambda+\mu) B_{1}^{3}}{\left[2 B_{1}(\lambda+\mu)^{2}+\left|B_{1}^{2}(2 \lambda+\mu)(\mu+1)-2 B_{2}(\lambda+\mu)^{2}\right|\right](2 \lambda+\mu)}, & B_{1}>\frac{(\lambda+\mu)^{2}}{2 \lambda+\mu} .\end{cases}
$$

and

$$
h(\lambda, \mu)= \begin{cases}\frac{B_{1}[(\mu+3)+|1-\mu|]}{2(2 \lambda+\mu)(\mu+1)}+\frac{2\left(\left|B_{2}\right|-B_{1}\right)}{(2 \lambda+\mu)(\mu+1)} & \left|B_{2}\right|>B_{1} \\ \frac{B_{1}[(\mu+3)+|1-\mu|]}{2(2 \lambda+\mu)(\mu+1)} & \left|B_{2}\right| \leq B_{1} .\end{cases}
$$

Proof. From equations (2.15) and (2.16) respectively for $n=2$ and $n=3$, we have that

$$
\begin{aligned}
& (\lambda+\mu) a_{2} b_{2}=B_{1} p_{1} \\
& (\mu+2 \lambda) a_{3} b_{3}+(\mu-1)\left(\lambda+\frac{\mu}{2}\right) a_{2}^{2} b_{2}^{2}=B_{1} p_{2}+B_{2} p_{1}^{2}, \\
& -(\lambda+\mu) a_{2} b_{2}=B_{1} q_{1}
\end{aligned}
$$

and

$$
-(\mu+2 \lambda) a_{3} b_{3}+(\mu+3)\left(\lambda+\frac{\mu}{2}\right) a_{2}^{2} b_{2}^{2}=B_{1} q_{2}+B_{2} q_{1}^{2} .
$$

From (3.6) and (3.8), we get

$$
p_{1}=-q_{1}
$$

By using (3.7), (3.9), and (3.10), it yields

$$
(2 \lambda+\mu)(\mu+1) a_{2}^{2} b_{2}^{2}=B_{1}\left(p_{2}+q_{2}\right)+2 B_{2} p_{1}^{2} .
$$

From (3.11) and (3.6), we have

$$
B_{1}^{2}(2 \lambda+\mu)(\mu+1) a_{2}^{2} b_{2}^{2}-2 B_{2}(\lambda+\mu)^{2} a_{2}^{2} b_{2}^{2}=B_{1}^{3}\left(p_{2}+q_{2}\right) .
$$

By (2.14) and (3.6), we get

$$
\begin{aligned}
\mid B_{1}^{2}(2 \lambda+\mu)(\mu+1) & -2 B_{2}(\lambda+\mu)^{2} b_{2}^{2}\left|a_{2}\right|^{2} \leq B_{1}^{3}\left(\left|p_{2}\right|+\left|q_{2}\right|\right) \\
\leq & 2 B_{1}^{3}\left(1-\left|p_{1}\right|^{2}\right)=2 B_{1}^{3}-2 B_{1}(\lambda+\mu)^{2} b_{2}^{2}\left|a_{2}\right|^{2} .
\end{aligned}
$$

Therefore,

$$
\left|a_{2}\right| \leq \frac{B_{1} \sqrt{2 B_{1}}}{\sqrt{\left[2 B_{1}(\lambda+\mu)^{2}+\left|B_{1}^{2}(2 \lambda+\mu)(\mu+1)-2 B_{2}(\lambda+\mu)^{2}\right|\right] b_{2}^{2}}}
$$

Also, by (2.14) and (3.11),

$$
\begin{aligned}
(2 \lambda+\mu)(\mu+1) b_{2}^{2}\left|a_{2}^{2}\right| & \leq B_{1}\left(\left|p_{2}\right|+\left|q_{2}\right|\right)+2\left|B_{2}\right|\left|p_{1}^{2}\right| \\
& \leq 2 B_{1}\left(1-\left|p_{1}\right|^{2}\right)+2\left|B_{2}\right|\left|p_{1}\right|^{2} \\
& =2 B_{1}+2\left|p_{1}\right|^{2}\left(\left|B_{2}\right|-B_{1}\right) \\
& \leq \begin{cases}2\left|B_{1}\right| & \left|B_{2}\right| \leq B_{1} \\
2\left|B_{2}\right| & \left|B_{2}\right|>B_{1} .\end{cases}
\end{aligned}
$$


Consequently,

$$
\left|a_{2}\right| \leq \begin{cases}\sqrt{\frac{2\left|B_{1}\right|}{(2 \lambda+\mu)(\mu+1) b_{2}^{2}}} & \left|B_{2}\right| \leq B_{1} \\ \sqrt{\frac{2\left|B_{2}\right|}{(2 \lambda+\mu)(\mu+1) b_{2}^{2}}} & \left|B_{2}\right|>B_{1} .\end{cases}
$$

Hence, the desired estimate on the coefficient $\left|a_{2}\right|$ as asserted in (3.4) is obtained from (3.12) and (3.13).

Next, in order to find the bound on the coefficient $\left|a_{3}\right|$, by subtracting (3.9) from (3.7), and using (3.10), we get

$$
2(2 \lambda+\mu) b_{3} a_{3}=B_{1}\left(p_{2}-q_{2}\right)+2(2 \lambda+\mu) a_{2}^{2} b_{2}^{2}
$$

From (2.14), (3.11) and (3.14), we have

$$
\begin{aligned}
b_{3} a_{3} & =\frac{B_{1}\left(p_{2}-q_{2}\right)}{2(2 \lambda+\mu)}+\frac{B_{1}\left(p_{2}+q_{2}\right)+2 B_{2} p_{1}^{2}}{(2 \lambda+\mu)(\mu+1)} \\
& =\frac{B_{1}\left[(\mu+3) p_{2}+(1-\mu) q_{2}\right]+4 B_{2} p_{1}^{2}}{2(2 \lambda+\mu)(\mu+1)}
\end{aligned}
$$

Therefore,

$$
\begin{aligned}
\left|a_{3}\right| & \leq \frac{B_{1}\left[(\mu+3)\left|p_{2}\right|+|1-\mu|\left|q_{2}\right|\right]+4\left|B_{2}\right|\left|p_{1}\right|^{2}}{2(2 \lambda+\mu)(\mu+1) b_{3}} \\
& \leq \frac{B_{1}\left[(\mu+3)\left(1-\left|p_{1}\right|^{2}\right)+|1-\mu|\left(1-\left|p_{1}\right|^{2}\right)\right]+4\left|B_{2}\right|\left|p_{1}\right|^{2}}{2(2 \lambda+\mu)(\mu+1) b_{3}} \\
& =\frac{B_{1}\left(1-\left|p_{1}\right|^{2}\right)[(\mu+3)+|1-\mu|]+4\left|B_{2}\right|\left|p_{1}\right|^{2}}{2(2 \lambda+\mu)(\mu+1) b_{3}} \\
& =\frac{B_{1}[(\mu+3)+|1-\mu|]+4\left|B_{2}\right|\left|p_{1}\right|^{2}-B_{1}\left|p_{1}\right|^{2}[(\mu+3)+|1-\mu|]}{2(2 \lambda+\mu)(\mu+1) b_{3}} .
\end{aligned}
$$

Thus for $\mu \geq 0$, we conclude that

$$
\begin{aligned}
\left|a_{3}\right| & \leq \frac{B_{1}[(\mu+3)+|1-\mu|]+4\left|B_{2}\right|\left|p_{1}\right|^{2}-4 B_{1}\left|p_{1}\right|^{2}}{2(2 \lambda+\mu)(\mu+1) b_{3}} \\
& =\frac{B_{1}[(\mu+3)+|1-\mu|]+4\left|p_{1}\right|^{2}\left(\left|B_{2}\right|-B_{1}\right)}{2(2 \lambda+\mu)(\mu+1) b_{3}} .
\end{aligned}
$$

Consequently,

$$
\left|a_{3}\right| \leq \frac{1}{b_{3}}\left\{\begin{array}{lr}
\frac{B_{1}[(\mu+3)+|1-\mu|]}{2(2 \lambda+\mu)(\mu+1)}+\frac{2\left(\left|B_{2}\right|-B_{1}\right)}{(2 \lambda+\mu)(\mu+1)} & \left|B_{2}\right|>B_{1} \\
\frac{B_{1}[(\mu+3)+|1-\mu|]}{2(2 \lambda+\mu)(\mu+1)} & \left|B_{2}\right| \leq B_{1} .
\end{array}\right.
$$


On the other hand, by using (2.14) and (3.10) for (3.14), we have

$$
\begin{aligned}
2(2 \lambda+\mu) b_{3}\left|a_{3}\right| & \leq B_{1}\left(\left|p_{2}\right|+\left|q_{2}\right|\right)+2(2 \lambda+\mu)\left|a_{2}\right|^{2} b_{2}^{2} \\
& \leq 2 B_{1}\left(1-\left|p_{1}\right|^{2}\right)+2(2 \lambda+\mu)\left|a_{2}\right|^{2} b_{2}^{2} .
\end{aligned}
$$

From (3.6), we get that

$$
B_{1}(2 \lambda+\mu) b_{3}\left|a_{3}\right| \leq\left[(2 \lambda+\mu) B_{1}-(\lambda+\mu)^{2}\right]\left|a_{2}\right|^{2} b_{2}^{2}+B_{1}^{2}
$$

Now, from (3.12), we have

$$
\begin{aligned}
B_{1}(2 \lambda+\mu) b_{3}\left|a_{3}\right| \leq & {\left[(2 \lambda+\mu) B_{1}-(\lambda+\mu)^{2}\right] } \\
& \times \frac{2 B_{1}^{3}}{2 B_{1}(\lambda+\mu)^{2}+\left|B_{1}^{2}(2 \lambda+\mu)(\mu+1)-2 B_{2}(\lambda+\mu)^{2}\right|}+B_{1}^{2} .
\end{aligned}
$$

Thus, we get

$$
\left|a_{3}\right| \leq \frac{B_{1}}{(2 \lambda+\mu) b_{3}}\left[1+\frac{2 B_{1}\left[B_{1}(2 \lambda+\mu)-(\lambda+\mu)^{2}\right]}{2 B_{1}(\lambda+\mu)^{2}+\left|B_{1}^{2}(\mu+1)(2 \lambda+\mu)-2 B_{2}(\lambda+\mu)^{2}\right|}\right] .
$$

Hence, from (3.17) and (3.18), we obtain the desired estimate on $\left|a_{3}\right|$ given in (3.5). This completes the proof.

Remark 3.6. Theorem 3.5 is an improvement of the estimates obtained by Bulut [8] in Theorem 2.2. It is easy to see that, for the coefficient $\left|a_{2}\right|$,

$$
\begin{gathered}
\frac{B_{1} \sqrt{2 B_{1}}}{\sqrt{\left[2 B_{1}(\lambda+\mu)^{2}+\left|B_{1}^{2}(2 \lambda+\mu)(\mu+1)-2 B_{2}(\lambda+\mu)^{2}\right|\right]}} \leq \frac{B_{1} \sqrt{2 B_{1}}}{\sqrt{2 B_{1}(\lambda+\mu)^{2}}} \\
=\frac{B_{1}}{\lambda+\mu} \\
\frac{B_{1} \sqrt{2 B_{1}}}{\sqrt{\left[2 B_{1}(\lambda+\mu)^{2}+\left|B_{1}^{2}(2 \lambda+\mu)(\mu+1)-2 B_{2}(\lambda+\mu)^{2}\right|\right]}} \\
\leq \frac{B_{1} \sqrt{2 B_{1}}}{\sqrt{\left|B_{1}^{2}(2 \lambda+\mu)(\mu+1)-2\left(B_{2}-B_{1}\right)(\lambda+\mu)^{2}\right|}}
\end{gathered}
$$

and if $\left|B_{2}\right| \leq B_{1}$ or $\left|B_{2}\right|>B_{1}$, then

$$
l(\lambda, \mu) \leq \sqrt{\frac{2\left(B_{1}+\left|B_{2}-B_{1}\right|\right)}{(2 \lambda+\mu)(\mu+1)}} .
$$

Also, for the coefficient $\left|a_{3}\right|$, we make the following cases

(i) If $B_{1} \leq \frac{(\lambda+\mu)^{2}}{2 \lambda+\mu}$, then

$$
\frac{B_{1}}{2 \lambda+\mu} \leq \frac{B_{1}}{2 \lambda+\mu}+\frac{2 B_{1}^{3}}{\sqrt{\left|B_{1}^{2}(2 \lambda+\mu)(\mu+1)-2\left(B_{2}-B_{1}\right)(\lambda+\mu)^{2}\right|}},
$$


and

$$
\frac{B_{1}}{2 \lambda+\mu} \leq \frac{B_{1}^{2}}{(\lambda+\mu)^{2}}+\frac{B_{1}}{2 \lambda+\mu}
$$

(ii) If $B_{1}>\frac{(\lambda+\mu)^{2}}{2 \lambda+\mu}$, then

$$
\begin{aligned}
& \frac{2 B_{1}^{3}(2 \lambda+\mu)+B_{1}\left|B_{1}^{2}(\mu+1)(2 \lambda+\mu)-2 B_{2}(\lambda+\mu)^{2}\right|}{(2 \lambda+\mu)\left[2 B_{1}(\lambda+\mu)^{2}+\left|B_{1}^{2}(\mu+1)(2 \lambda+\mu)-2 B_{2}(\lambda+\mu)^{2}\right|\right]} \\
= & \frac{2 B_{1}^{3}}{2 B_{1}(\lambda+\mu)^{2}+\left|B_{1}^{2}(\mu+1)(2 \lambda+\mu)-2 B_{2}(\lambda+\mu)^{2}\right|} \\
& +\frac{B_{1}\left|B_{1}^{2}(\mu+1)(2 \lambda+\mu)-2 B_{2}(\lambda+\mu)^{2}\right|}{(2 \lambda+\mu)\left[2 B_{1}(\lambda+\mu)^{2}+\left|B_{1}^{2}(\mu+1)(2 \lambda+\mu)-2 B_{2}(\lambda+\mu)^{2}\right|\right]} \\
\leq & \frac{2 B_{1}^{3}}{2 B_{1}(\lambda+\mu)^{2}}+\frac{B_{1}\left|B_{1}^{2}(2 \lambda+\mu)(\mu+1)-2 B_{2}(\lambda+\mu)^{2}\right|}{(2 \lambda+\mu)\left|B_{1}^{2}(\mu+1)(2 \lambda+\mu)-2 B_{2}(\lambda+\mu)^{2}\right|} \\
\leq & \frac{B_{1}^{2}}{(\lambda+\mu)^{2}}+\frac{B_{1}}{2 \lambda+\mu} .
\end{aligned}
$$

(iii) If $\left|B_{2}\right| \leq B_{1}$ or $\left|B_{2}\right|>B_{1}$, then

$$
h(\lambda, \mu) \leq \frac{B_{1}[(\mu+3)+|1-\mu|]}{2(\mu+1)(2 \lambda+\mu)}+\frac{2\left|B_{2}-B_{1}\right|}{(2 \lambda+\mu)(\mu+1)} .
$$

Remark 3.7. With similar argument of Remark 3.6 in Theorem 3.5,

(1) If we take $\Theta(z)=\frac{z}{1-z}$, then we obtain improvement of the estimates obtained by Tang et al. [25, Theorem 2.1].

(2) If we set $\lambda=\mu=1$ and $\Theta(z)=\frac{z}{1-z}$, we get improvement of the estimates obtained by Ali et al. [4, Theorem 2.1].

(3) By taking $\mu=1, \lambda \geq 1$ and $\Theta(z)=\frac{z}{1-z}$, we obtain improvement of the estimates obtained by Kumar et al. [20, Theorem 2.2].

Taking $\varphi(z)=\left(\frac{1+z}{1-z}\right)^{\alpha}$ in Theorem 3.5, then we have the following result.

Corollary 3.8. Let $f \in \mathcal{H}_{\Sigma}^{\lambda, \mu}(\varphi ; \Theta)$ be given by (1.1), $\lambda \geq 1$ and $\mu \geq 0$. Then

$$
\left|a_{2}\right| \leq \frac{1}{b_{2}} \min \left\{\sqrt{\frac{4 \alpha}{(2 \lambda+\mu)(\mu+1)}}, \frac{2 \alpha}{\sqrt{(\lambda+\mu)^{2}+\alpha\left|\mu+2 \lambda-\lambda^{2}\right|}}\right\}
$$

and

$$
\left|a_{3}\right| \leq \frac{1}{b_{3}} \min \left\{k(\lambda, \mu), \frac{\alpha[(\mu+3)+|1-\mu|]}{(2 \lambda+\mu)(\mu+1)}\right\}
$$


where

$$
k(\lambda, \mu)=\left\{\begin{array}{lr}
\frac{2 \alpha}{(2 \lambda+\mu)}, & 0<\alpha \leq \frac{(\lambda+\mu)^{2}}{2(2 \lambda+\mu)} \\
\frac{2 \alpha^{2}\left|\mu+2 \lambda-\lambda^{2}\right|+4 \alpha^{2}(2 \lambda+\mu)}{\left[(\lambda+\mu)^{2}+\alpha\left|\mu+2 \lambda-\lambda^{2}\right|\right](2 \lambda+\mu)}, & \frac{(\lambda+\mu)^{2}}{2(2 \lambda+\mu)}<\alpha \leq 1 .
\end{array}\right.
$$

Remark 3.9. With similar argument of Remark 3.6 in Corollary 3.8,

(1) If $\mu=1$, then we have improvement of the estimates which were given by El-Ashwah [11, Theorem 1].

(2) If $\lambda=1$ and $\Theta(z)=\frac{z}{1-z}$, then we have improvement of the estimates which were given by Prema and Keerthi [23, Theorem 2.2].

(3) If $\Theta(z)=\frac{z}{1-z}$, then we obtain the same estimate $\left|a_{3}\right|$ and improvement of the estimates which were given by Çăglar et al. [9, Theorem 2.2].

(4) If $\mu=\lambda=1$ and $\Theta(z)=\frac{z}{1-z}$, then we have improvement of the estimates which were given by Srivastava et al, [24, Theorem 1].

(5) If $\mu=1$ and $\Theta(z)=\frac{z}{1-z}$, then we have improvement of the estimates which were given by Frasin and Aouf [12, Theorem 2.2].

(6) If $\mu=1$ and $\Theta(z)=z+\sum_{n=2}^{\infty} \frac{\left(a_{1}\right)_{n-1} \cdots\left(a_{q}\right)_{n-1}}{\left(b_{1}\right)_{n-1} \cdots\left(b_{s}\right)_{n-1}} \frac{1}{n !} z^{n}$, then we have improvement of the estimates which were given by Aouf et al. [5, Theorem 4].

(7) If $\lambda=1, \mu=0$ and $\Theta(z)=\frac{z}{1-z}$, then we have improvement of the estimates which were given by Brannan and Taha [7].

By choosing $\varphi(z)=\frac{1+(1-2 \beta) z}{1-z}$, in Theorem 3.1, then we have the following result.

Corollary 3.10. Let $f \in \mathcal{H}_{\Sigma}^{\lambda, \mu}(\varphi ; \Theta)$ be given by $(1.1), \lambda \geq 1$ and $\mu \geq 0$. Then

$$
\begin{aligned}
\left|a_{2}\right| \leq \frac{1}{b_{2}} \min \left\{\sqrt{\frac{4(1-\beta)}{(2 \lambda+\mu)(\mu+1)^{\prime}}}\right. & \left.\frac{2(1-\beta)}{\sqrt{(\lambda+\mu)^{2}+\left|(1-\beta)(2 \lambda+\mu)(\mu+1)-(\lambda+\mu)^{2}\right|}}\right\},
\end{aligned}
$$

and

$$
\left|a_{3}\right| \leq \frac{1}{b_{3}} \min \left\{k(\lambda, \mu), \frac{(1-\beta)[(\mu+3)+|1-\mu|]}{2(2 \lambda+\mu)(\mu+1)}\right\}
$$

where

$$
k(\lambda, \mu)= \begin{cases}\frac{2(1-\beta)}{(2 \lambda+\mu)}, & \frac{2(2 \lambda+\mu)-(\lambda+\mu)^{2}}{2(2 \lambda+\mu)} \leq \beta<1 \\ \frac{2(1-\beta)\left|(1-\beta)(2 \lambda+\mu)(\mu+1)-(\lambda+\mu)^{2}\right|+4(2 \lambda+\mu)(1-\beta)^{2}}{\left[(\lambda+\mu)^{2}+\left|(1-\beta)(2 \lambda+\mu)(\mu+1)-(\lambda+\mu)^{2}\right|\right](2 \lambda+\mu)}, & 0 \leq \beta<\frac{2(2 \lambda+\mu)-(\lambda+\mu)^{2}}{2(2 \lambda+\mu)} .\end{cases}
$$


Remark 3.11. With similar argument of Remark 3.6, in Corollary 3.10,

(1) If $\mu=1$, then we have improvement of the estimates which were given by El-Ashwah [11, Theorem 2].

(2) If $\lambda=1$ and $\Theta(z)=\frac{z}{1-z}$, then we have improvement of the estimates which were given by Prema and Keerthi [23, Theorem 3.2].

(3) If $\Theta(z)=\frac{z}{1-z}$, then we obtain the same estimate of $\left|a_{3}\right|$ and improvement of the estimates which were given by Çağlar et al. [9, Theorem 3.1].

(4) If $\mu=\lambda=1$ and $\Theta(z)=\frac{z}{1-z}$, then we have improvement of the estimates which were given by Srivastava et al, [24, Theorem 2].

(5) If $\mu=1$ and $\Theta(z)=\frac{z}{1-z}$, then we have improvement of the estimates which were given by Frasin and Aouf [12, Theorem 3.2].

(6) If $\mu=1$ and $\Theta(z)=z+\sum_{n=2}^{\infty} \frac{\left(a_{1}\right)_{n-1} \cdots\left(a_{q}\right)_{n-1}}{\left(b_{1}\right)_{n-1} \cdots\left(b_{s}\right)_{n-1}} \frac{1}{n !} z^{n}$, then we have improvement of the estimates which were given by Aouf et al. [5, Theorem 8].

(7) If $\lambda=1, \mu=0$ and $\Theta(z)=\frac{z}{1-z}$, then we have improvement of the estimates which were given by Brannan and Taha [7].

(8) If $\mu=1$ and $\Theta(z)=\frac{z}{1-z}$, then we have improvement of the estimates which were given by Jahangiri et al. [16, Theorem 2].

(9) If $\Theta(z)=\frac{z}{1-z}$, then we have improvement of the estimates which were given by Bulut [8, Theorem 2].

(10) If $\Theta(z)=\frac{z}{1-z}$, then we have improvement of the estimates which were given by Jahangiri et al. [18, Theorem 2.2].

\section{Acknowledgment}

The authors wish to sincerely thank the referee, for the careful reading of the paper and for the helpful suggestions and comments.

\section{References}

[1] H. Airault, Remarks on Faber polynomials, Int. Math. Forum., 3 (2008) 449456.

[2] H. Airault and A. Bouali, Differential calculus on the Faber polynomials, Bull. Sci. Math., 130 (2006) 179-222.

[3] H. Airault and J. Ren, An algebra of differential operators and generating functions on the set of univalent functions, Bull. Sci. Math., 126 (2002) 343367. 
[4] R. M. Ali, S. K. Lee, V. Ravichandran and S. Subramaniam, Coefficient estimates for bi-univalent Ma-Minda starlike and convex functions, Appl. Math. Lett., 25 (2012) 344-351.

[5] M. K. Aouf, R. M. El-Ashwah, and A. M. Abd-Eltawab, New subclasses of biunivalent functions involving Dziok-Srivastava operator, ISRN Math. Anal., (2013) Art. ID 387178, 5 Pages.

[6] D. A. Brannan and J. G. Clunie (Eds.), Aspects of Contemporary Complex Analysis Proceedings of the NATO Advanced Study Institute held at the University of Durham, Durham; July 1-20, (1979) (Academic Press, New York and London, 1980).

[7] D. A. Brannan and T. S. Taha, On some classes of bi-univalent functions, Studia Univ. Babeş-Bolyai Math., 31 (2) (1986) 70-77.

[8] S. Bulut, Coefficient estimates for a new subclass of analytic and bi-univalent functions defined by Hadamard product. J. Complex Anal., (2014) Art. ID 302019, 7 Pages.

[9] M. Çă̆lar, H, Orhan and N. Yağmur, Coefficient bounds for new subclasses of bi-univalent functions, Filomat., 27 (2013) 1165-1171.

[10] P. L. Duren, Univalent Functions, Grundlehren der Mathematischen Wissenschaften, Band 259, Springer-Verlag, New York, Berlin, Heidelberg and Tokyo, 1983.

[11] R. M. El-Ashwah, Subclasses of bi-univalent functions defined by convolution, J. Egyptian Math. Soc., 22 (3) (2014) 348-351.

[12] B. A. Frasin and M. K. Aouf, New subclasses of bi-univalent functions, Appl. Math. Lett., 24 (2011) 1569-1573.

[13] G. Faber, Über polynomische Entwickelungen, Math. Ann., 57 (3) (1903) 389408.

[14] S. G. Hamidi, S. A. Halim and J. M. Jahangiri, Faber polynomial coefficient estimates for meromorphic bi-starlike functions, Int. J. Math. Math. Sci., (2013) Art. ID 498159, 4 pages.

[15] S. G. Hamidi, S. A. Halim and J. M. Jahangiri, Coefficient estimates for a class of meromorphic bi-univalent functions, C. R. Math. Acad. Sci. Paris, Ser. I., 351 (2013) 349-352.

[16] J. M. Jahangiri and S. G. Hamidi, Coefficient estimates for certain classes of bi-univalent functions, Int. J. Math. Math. Sci., (2013) Art. ID 190560, 4 pages.

[17] J. M . Jahangiri, S .G. Hamidi and S. A. Halim, Coefficients of bi-univalent functions with positive real part derivatives, Bull. Malays. Math. Sci. Soc., 37 (2014) 633-640. 
[18] J. M . Jahangiri and S .G .Hamidi, Faber polynomial coefficient estimates for analytic bi-Bazilevič functions, Matematicki Vesnik., 67 (2015) 123-129.

[19] A. W. Kedzierawski, Some remarks on bi-univalent functions, Ann. Univ. Mariae Curie-Sk lodowska Sect. A., 39 (1985) 77-81.

[20] S. S. Kumar, V. Kumar and V. Ravichandran, Estimates for the initial coefficients of bi-univalent functions, Tamsui Oxf. J. Math. Sci., 29 (2013) 487-504.

[21] M. Lewin, On a coefficient problem for bi-univalent functions, Proc. Amer. Math. Soc., 18 (1967) 63-68.

[22] Z. Nehari, Conformal mapping, McGraw-Hill, New York, 1952.

[23] S. Prema and B. S. Keerthi, Coefficient bounds for certain subclasses of analytic functions, J. Math. Anal., 4 (2013) 22-27.

[24] H. M. Srivastava, A. K. Mishra and P. Gochhayat, Certain subclasses of analytic and biunivalent functions, Appl. Math. Lett., 23 (2010) 1188-1192.

[25] H. Tang, G.-T. Deng, and S.-H. Li, Coefficient estimates for new subclasses of Ma-Minda bi-univalent functions. J. Inequal. Appl., (2013) Art. 317, 10 Pages.

[26] P. G. Todorov, On the Faber polynomials of the univalent functions of class $\Sigma$, J. Math. Anal. Appl., 162 (1991) 268-276.

Department of Mathematics, Shahrood University of Technology,

P.O.Box 316-36155, Shahrood, Iran

emails: azireh@gmail.com, e_analoei@ymail.com

Kocaeli University, Faculty of Aviation and Space Sciences,

Arslanbey Campus,

41285 Kartepe, Kocaeli, Turkey

e-mail: serap.bulut@kocaeli.edu.tr 